Correspondence

Sabine Gröbner

sabine.groebner@

med.uni-tuebingen.de

Received 13 August 2008

Accepted 10 March 2009

\section{Emergence of carbapenem-non-susceptible extended-spectrum $\beta$-lactamase-producing Klebsiella pneumoniae isolates at the university hospital of Tübingen, Germany}

\author{
Sabine Gröbner, ${ }^{1}$ Dirk Linke, ${ }^{2}$ Wolfgang Schütz, ${ }^{3}$ Claudia Fladerer, ${ }^{3}$ \\ Johannes Madlung, ${ }^{3}$ Ingo B. Autenrieth, ${ }^{1}$ Wolfgang Witte ${ }^{4}$ \\ and Yvonne Pfeifer ${ }^{4}$
}

${ }^{1}$ Institute of Medical Microbiology and Hygiene, Eberhard Karls University of Tübingen, ElfriedeAulhorn-Str. 6, D-72076 Tübingen, Germany

${ }^{2}$ Max Planck Institute for Developmental Biology, Department I, Protein Evolution, Spemannstr. 35, D-72076 Tübingen, Germany

${ }^{3}$ Proteome Centre Tübingen, Interfaculty Institute of Cell Biology, University of Tübingen, Auf der Morgenstelle 15, D-72076 Tübingen, Germany

${ }^{4}$ Robert Koch Institute, Burgstr. 37, D-38855 Wernigerode, Germany

The spread of Gram-negative bacteria with plasmid-borne extended-spectrum $\beta$-lactamases (ESBLs) has become a worldwide problem. This study analysed a total of 366 ESBL-producing Enterobacteriaceae strains isolated from non-selected patient specimens at the university hospital of Tübingen in the period January 2003 to December 2007. Although the overall ESBL rate was comparatively low (1.6\%), the percentages of ESBL-producing Enterobacter spp. and Escherichia coli increased from 0.8 and $0.5 \%$, respectively, in 2003 to 4.6 and $3.8 \%$ in 2007 . In particular, the emergence was observed of one carbapenem-resistant ESBL-producing E. coli isolate and five carbapenem-non-susceptible ESBL-positive Klebsiella pneumoniae isolates, in two of which carbapenem resistance development was documented in vivo under a meropenemcontaining antibiotic regime. The possible underlying mechanism for this carbapenem resistance in three of the K. pneumoniae isolates was loss of the Klebsiella porin channel protein OmpK36 as shown by PCR analysis. The remaining two $K$. pneumoniae isolates exhibited increased expression of a tripartite AcrAB-TolC efflux pump as demonstrated by SDS-PAGE and mass spectrometry analysis of bacterial outer-membrane extracts, which, in addition to other unknown mechanisms, may contribute towards increasing the carbapenem MIC values further.

Carbapenem-non-susceptible ESBL isolates may pose a new problem in the future due to possible outbreak situations and limited antibiotic treatment options. Therefore, a systematic exploration of intestinal colonization with ESBL isolates should be reconsidered, at least for haemato-oncological departments from where four of the five carbapenem-non-susceptible ESBL isolates originated.

\section{INTRODUCTION}

In contrast to the USA and the Western Pacific area, the prevalence of extended-spectrum $\beta$-lactamases (ESBLs) in Germany was comparably low in 2001 (8.2\% of Klebsiella pneumoniae and $0.8 \%$ of Escherichia coli isolates; Sturenburg \& Mack, 2003). However, the prevalence of ESBL-producing Gram-negative bacteria is increasing, both

Abbreviations: ESBL, extended-spectrum $\beta$-lactamase; FCCP, trifluoromethoxyphenylhydrazone; PA $\beta \mathrm{N}$, phenylalanine arginine $\beta$-naphthylamide. in hospitals and in the community (Sturenburg \& Mack, 2003; Pitout et al., 2005).

ESBLs confer resistance to amino- and ureidopenicillins, oximino-cephalosporins and monobactams. As co-resistance to non- $\beta$-lactam antimicrobials is common among ESBL isolates, the treatment options are limited. Among the E. coli and K. pneumoniae isolates predominantly found to produce ESBLs in Europe, carbapenem-non-susceptible isolates are becoming more widespread (MartínezMartínez et al., 1999; Mena et al., 2006). The underlying 
mechanism for carbapenem resistance of ESBL-positive $K$. pneumoniae isolates is loss of porin channels (OmpK35 and/or OmpK36) for antibiotic uptake (Martínez-Martínez et al., 1999) combined with the expression of AmpC $\beta$ lactamases (Bradford et al., 1997) or expression of carbapenemases (Woodford et al., 2004).

The present study was conducted to evaluate the development of ESBL-producing isolates at our university hospital between 2003 and 2007. We also analysed the mechanisms of resistance and epidemiology of five carbapenem-non-susceptible K. pneumoniae isolates found during this study.

\section{METHODS}

Collection and phenotypic detection of ESBL-positive strains. From January 2003 to December 2007, 366 non-duplicated ESBLproducing isolates were collected from non-selected patient samples in our tertiary-care hospital in the south-western part of Germany. The hospital has 1500 beds and treats approximately 68000 inpatients and 220000 outpatients per year. Enterobacteriaceae isolates were identified by using API 20 E or Vitek2 (bioMérieux). Susceptibility testing was performed by using Vitek2 or agar diffusion following the Clinical and Laboratory Standards Institute guidelines. For the detection of ESBLs, combination disc testing was performed with cefpodoxime (January 2003-August 2004 and February 2007December 2007), ceftazidime and cefotaxime discs and their clavulanate-containing counterparts (January 2003-December 2007), and by ESBL combination disc testing with cefepime and its clavulanate-containing counterpart (AB Biodisk and Mast Diagnostica) (September 2004-December 2007). To confirm mer- openem resistance, a microbroth dilution assay and meropenem Etest (AB Biodisk) were performed.

Phenotypic identification of ESBL producers that were resistant or intermediate to carbapenems was performed in a second, confirmatory microbroth dilution test detecting resistance to three thirdgeneration cephalosporins (cefotaxime, ceftazidime and cefpodoxime) with and without clavulanic acid (CLSI, 2008). If the MIC of cefpodoxime was $\geqslant 8 \mu \mathrm{g} \mathrm{ml}^{-1}$ and the MIC of ceftazidime and/or cefotaxime was $\geqslant 2 \mu \mathrm{g} \mathrm{ml}^{-1}$, and, in addition, if the MIC ratio of an oximino-cephalosporin with and without inhibitor was $\geqslant 8$, the strain was inferred to be an ESBL producer (Sturenburg \& Mack, 2003). The presence of metallo- $\beta$-lactamases was tested by using Etest MBL (AB Biodisk) (Walsh et al., 2002).

PCR and sequence analysis of resistance genes. The genes for ESBLs of the carbapenem-intermediate and -resistant ESBL producers (bla $a_{\mathrm{TEM}}, b l a_{\mathrm{SHV}}$ and $\left.b l a_{\mathrm{CTX}-\mathrm{M}}\right)$ were amplified by using multiplex PCR. The presence of relevant carbapenem-resistance genes (bla $a_{\mathrm{KPC}}$, $b l a_{\mathrm{OXA}-23}, b l a_{\mathrm{OXA}-24}, b l a_{\mathrm{OXA}-48}, b l a_{\mathrm{OXA}-58}, b l a_{\mathrm{VIM}}$ and $\left.b l a_{\mathrm{IMP}}\right)$ was investigated by using the primers given in Table 1. PCR for detection of $b l a_{\mathrm{GIM}}, b l a_{\mathrm{SIM}}$ and $b l a_{\mathrm{SPM}}$ genes was carried out with primer pairs described previously (Mendes et al., 2007). Intrinsic and acquired $\operatorname{ampC}$ genes in cefoxitin-resistant isolates were investigated by using a multiplex PCR that differentiated between the six gene families of plasmid-borne ampC and their chromosome-encoded progenitor genes (Pérez-Pérez \& Hanson, 2002). Furthermore, the ampC promoter region of the cefoxitin-resistant, carbapenem-non-susceptible E. coli isolate was sequenced as described previously (Mulvey et al., 2005). Sequencing of all amplicons was carried out by using an ABI PRISM BigDye 3.1 Terminator Cycle Sequencing Ready Reaction kit (Applied Biosystems).

Resistance gene transfer and plasmid analysis. Transfer of third-generation cephalosporin and carbapenem resistance was tested

Table 1. Primer sequences for detection of different $\beta$-lactamase genes

\begin{tabular}{|c|c|c|c|c|c|}
\hline Primer name & Sense/antisense & Sequence $\left(5^{\prime} \rightarrow 3^{\prime}\right)$ & Target & Product length $(b p)$ & Source \\
\hline TEMu & Antisense & TTAATCAGTGAGGCACCTAT & & & \\
\hline SHVu & Antisense & GGTTAGCGTTGCCAGTGCT & & & \\
\hline CTX-Mu & Sense & CGCTTTGCGATGTGCAG & All bla ${ }_{\mathrm{CTX}-\mathrm{M}}$ genes & 551 & Paterson et al. (2003) \\
\hline CTX-Mu & Antisense & ACCGCGATATCGTTGGT & & & \\
\hline CTX-M9 & Antisense & TATCATTGGTGGTGCCGTAG & & & \\
\hline OXA-23u & Sense & AACCCCGAGTCAGATTGTTC & $b l a_{\text {OXA-23-related genes }}$ & 642 & This study \\
\hline OXA-23u & Antisense & ATTTTTCCATCTGGCTGCTC & & & \\
\hline OXA-24u & Sense & GTTTCTCTCAGTGCATGTTCATC & $b l a_{\text {OXA-24}}$-related genes & 740 & This study \\
\hline OXA-24u & Antisense & CGAATAGAACCAGACATTCCTTC & & & \\
\hline OXA-48u & Sense & AAATCACAGGGCGTAGTTGTG & $b l a_{\mathrm{OXA}-48}$-related genes & 555 & This study \\
\hline $\mathrm{IMPu}$ & Antisense & ATAATTTGGCGGACTTTGGC & & & \\
\hline VIMu & Sense & AGTGGTGAGTATCCGACAG & All $b l a_{\mathrm{VIM}}$ genes & 261 & This study \\
\hline VIMu & Antisense & ATGAAAGTGCGTGGAGAC & & & \\
\hline $\mathrm{KPCu}$ & Sense & CAGCTCATTCAAGGGCTTTC & All $b l a_{\mathrm{KPC}}$ genes & 533 & This study \\
\hline $\mathrm{KPCu}$ & Antisense & AGTCATTTGCCGTGCCATAC & & & \\
\hline
\end{tabular}


by broth mating assays using a sodium azide-resistant E. coli J53 recipient. Selection of transconjugants was performed on MuellerHinton agar plates containing sodium azide $\left(200 \mu \mathrm{g} \mathrm{ml}^{-1}\right)$, ampicillin $\left(100 \mu \mathrm{g} \mathrm{ml}^{-1}\right)$ and meropenem $\left(2 \mu \mathrm{g} \mathrm{ml}^{-1}\right)$. The plasmid DNA of the donor and transconjugants was isolated with a Plasmid Mini kit (Qiagen) and analysed on a $0.4 \%$ agarose gel using E. coli V517 as a size marker (Macrina et al., 1978).

For Southern blot hybridization, specific probes for $b l a_{\mathrm{TEM}}, b l a_{\mathrm{SHV}}$ and $b l a_{\text {CTX-M }}$ were labelled with a PCR DIG Labelling mix (Roche Diagnostics) using DIG-DNA Ladder III (Roche) as a size marker.

Investigation of porins. To determine the presence or absence of the two major porins (outer-membrane proteins) of $K$. pneumoniae, OmpK35 and OmpK36, the gene fragments of ompK35 and ompK36 of the carbapenem-non-susceptible $K$. pneumoniae ESBL producers were amplified and sequenced by using primers described previously (Lee et al., 2007).

Analysis of outer-membrane protein profiles by SDS-PAGE and MS. The carbapenem-non-susceptible $K$. pneumoniae isolates and, as a control, several E. coli BL21(DE3) mutant strains (Prilipov et al., 1998) were cultivated overnight in Luria broth at $37^{\circ} \mathrm{C}$, diluted $(1: 20)$ in fresh medium and incubated for a further $2 \mathrm{~h}$. The outer membranes were isolated from cultures as described previously (Osborn et al., 1972), washed three times with water, added to SDSPAGE sample buffer and subjected to SDS-PAGE (11\% polyacrylamide, $8 \mathrm{M}$ urea) according to a method described previously with some modifications (Schägger \& von Jagow, 1987).

The gels were stained overnight with Coomassie solution $(0.25 \%$ Coomassie R250; $8 \%$, v/v, acetic acid; $40 \%$, v/v, methanol) at $4{ }^{\circ} \mathrm{C}$, and then destained for $1-2 \mathrm{~h}$ with $10 \%(\mathrm{v} / \mathrm{v})$ acetic acid, $40 \%(\mathrm{v} / \mathrm{v})$ ethanol. Slices containing the proteins of interest were cut out and ingel protein digestions were carried out overnight at $37{ }^{\circ} \mathrm{C}$ with trypsin (porcine, sequencing grade, modified; Promega).

Reversed-phase nano-liquid chromatography tandem MS was performed as described elsewhere (Liu et al., 2006). In brief, the tryptic peptide mixture was separated using an Ultimate nanoflow LC system (Dionex/LC Packings) coupled to a QSTAR Pulsar I hybrid QqTOF mass spectrometer (Applied Biosystems/MDS Sciex), equipped with a nano-electrospray ion source (New Objective).

Proteins were identified by correlating the data from the MS/MS spectra with the NCBI.nr protein sequence database (version 20080206, taxonomy bacteria) using the MOWSE algorithm as implemented in the search engine MASCOT (Matrix Science; Perkins et al., 1999). All experimental data obtained by gel electrophoresis and MS were stored in a LIMS database (Proteinscape 1.3; Bruker Daltonics). Database search results were reviewed as described elsewhere (Barjaktarovic et al., 2007) with the following modification according to specific database characteristics: individual MASCOT ion scores $>49$ indicated identity or extensive homology.

Statistical analysis. Hybase software (Cymed AG) was used for statistical analysis of the microbiological findings.

\section{RESULTS AND DISCUSSION}

\section{Development and epidemiology of ESBL isolates from 2003 to 2007}

From January 2003 to December 2007, 366 non-duplicated ESBL-producing isolates were collected from non-selected patient samples. Fig. 1(a) shows that, among these, E. coli (a)

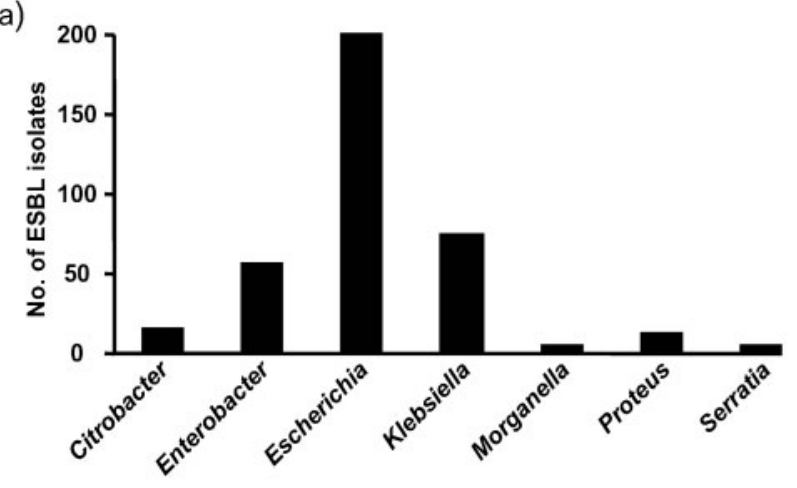

(b)

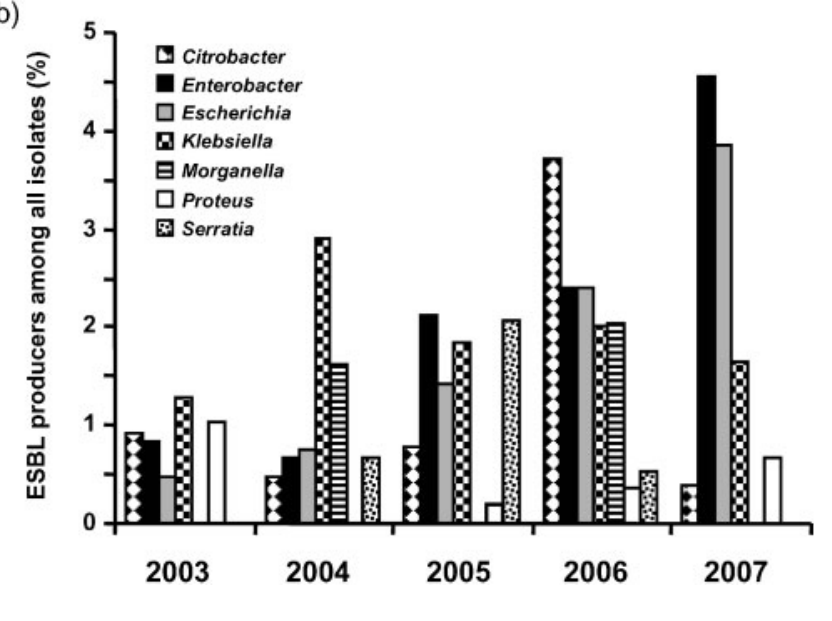

Fig. 1. ESBL isolates 2003-2007 ( $n=366$ ). (a) Total number of ESBL isolates per species. The corresponding organisms were Citrobacter amalonaticus $(n=1)$, Citrobacter braakii $(n=2)$, Citrobacter farmeri $(n=1)$, Citrobacter freundii $(n=9)$, Citrobacter koseri $(n=2)$, Enterobacter aerogenes $(n=13)$, Enterobacter cloacae $(n=43)$, Escherichia coli $(n=200)$, Klebsiella ornithinolytica $(n=4)$, Klebsiella oxytoca $(n=3)$, Klebsiella pneumoniae $(n=66)$, Klebsiella terrigena $(n=1)$, Morganella morganii $(n=4)$, Proteus mirabilis $(n=10)$, Proteus penneri $(n=1)$, Proteus vulgaris $(n=1)$, Serratia fonticola $(n=4)$ and Serratia marcescens $(n=1)$. (b) The percentage of ESBL isolates per species with respect to the overall number of isolates (ESBL-positive and -negative) of the corresponding species.

was the most frequent $(n=200)$, followed by Klebsiella spp. $(n=74)$, Enterobacter spp. $(n=56)$ and other species (Citrobacter spp., $n=15$; Proteus spp., $n=12$; Serratia spp., $n=5$; Morganella spp., $n=4)$. When the number of ESBL-positive isolates was compared with the overall number of isolates of the corresponding bacterial species (Fig. 1b), it became obvious that the rate of ESBL-positive Enterobacter spp. and E. coli isolates continuously increased from 0.8 and $0.5 \%$, respectively, in 2003 to 4.6 and $3.8 \%$, respectively, in 2007. Although an overall increase in ESBLpositive E. coli isolates was observed from 2003 to 2007, on average the E. coli ESBL rate was low (1.8\%), which is comparable to the low rate of $0.8 \%$ ESBL-producing E. coli 
isolates reported for 2001 in Germany (Sturenburg \& Mack, 2003). The percentage of ESBL-positive Klebsiella strains in our hospital was much lower (between 1.3 and $2.9 \%$ ) compared with the Germany-wide rate of $8.2 \%$ reported in 2001 (Sturenburg \& Mack, 2003).

The results of studies determining whether ESBL production has an effect on clinical outcomes are divergent (Ramphal \& Ambrose, 2006). Nevertheless, at least $62.8 \%$ of the ESBL producers isolated in our hospital caused infections, as they were isolated from urine samples exhibiting bacterial monoculture and high bacterial counts $\left(>10^{4} \mathrm{ml}^{-1}\right.$; $42.1 \%)$, intraoperative swabs and punctates $(12.8 \%)$, blood $(4.9 \%)$, bronchoalveolar lavages $(1.6 \%)$ and other relevant specimens $(1.4 \%)$. The fact that most ESBL producers $(42.1 \%)$ were cultivated from urine is in accordance with a study performed at a Croatian university hospital reporting that the most abundant source of ESBL-producing strains were urinary-tract infections (Tonkic et al., 2005). Of the ESBL-producing isolates, $16.4 \%$ were derived from wounds. However, it is difficult to distinguish between infection and colonization of wounds, as the isolation of ESBL producers from deep wounds such as bedsores or ulcers does not necessarily indicate infection. By contrast, $20.8 \%$ of the ESBL isolates were considered to have colonized patients without infection, as they were isolated from the upper respiratory tract $(13.7 \%)$, vaginal swabs $(3.8 \%)$, and stools and anal or rectal swabs $(3.3 \%)$. Compared with a recent study reporting a rate of $8.1 \%$ faecal carriage of ESBLproducing Enterobacteriaceae in hospitalized patients (Castillo García et al., 2007), the rate of faecal ESBL carriage at our clinic was low (3.3\%). Hence, we assume that, in our hospital, a considerable number of patients carrying faecal ESBL producers remain undetected, as screening for faecal carriage of ESBL producers has not been implemented in our hospital to date.

Most of the ESBL producers were isolated from patients from intensive care units $(17.8 \%)$, internal medicine departments $(16.9 \%)$ and surgery departments $(11.2 \%)$. A considerable number of ESBL-producing strains (23.8\%) were isolated from patients admitted to one of the four external hospitals that are associated with our university hospital (all a maximum of $20 \mathrm{~km}$ from the university hospital). Although there is no definite consensus on the hospital care of patients carrying or being infected with ESBL-producing bacteria, the management of ESBLpositive patients at our hospital follows uniform hospitalwide guidelines based on generally accepted recommendations such as those from the Robert Koch Institute on a national level and the Centers for Disease Control and Prevention on an international level to prevent the transmission of bacteria.

In spite of strict hygiene management, we observed an increase in ESBL-positive isolates from 2003 to 2007, in particular Enterobacter spp. and E. coli, at our hospital. As there was no suggestion of a possible outbreak caused by ESBL-positive organisms, it cannot be excluded that community-acquired ESBL producers were increasingly imported to our hospital. Several studies reporting the influx of ESBL-producing organisms into the hospital suggest that ESBL-producing Enterobacteriaceae should be considered not only as nosocomial pathogens but also as community-acquired pathogens (Ben-Ami et al., 2006; Oteo et al., 2006; Pitout et al., 2005). Recently, it has been reported that even household contacts of community ESBL patients lead to high rates of intestinal colonization of the contact persons (16.7\%) (Valverde et al., 2008). Unfortunately, in our hospital, the rate of patients who carried ESBL-producing bacteria at admission could not be evaluated, as patients were not routinely screened for ESBLs when they were admitted to the hospital.

\section{Antimicrobial susceptibility profiles of the ESBL producers}

The occurrence of ESBLs in members of the Enterobacteriaceae results in resistance to many different $\beta$ lactam antibiotics. The prevalent location of ESBL genes and further resistance genes on conjugative plasmids leads to coresistance to non- $\beta$-lactam antibiotics and the transmission of resistance among enterobacterial species. Antimicrobial testing of the ESBL producers isolated in our hospital revealed that $83.9 \%(47 / 56)$ of Enterobacter, $73.3 \%(55 / 75)$ of Klebsiella, $72.7 \%$ (8/11) of Proteus and $60.0 \%$ (9/15) of Citrobacter species were susceptible to levofloxacin, and $80.0 \%(4 / 5)$ of Serratia, $63.6 \%$ (7/11) of Proteus and 53.3\% $(8 / 15)$ of Citrobacter species were susceptible to cotrimoxazole (Fig. 2), providing antibiotic treatment options other than carbapenems for patients infected with these bacteria. Detailed analysis of the 102 ESBL-producing E. coli isolated from urine revealed that co-resistance to non- $\beta$-lactam antibiotics was frequent among these isolates $[64.7 \%(66 /$ 102) to cotrimoxazole and $76.4 \%(78 / 102)$ to levofloxacin] when compared with the overall $27.6 \%$ (1103/3990) cotrimoxazole-resistant and $14.4 \%$ (576/3990) levofloxacin-resistant non-ESBL E. coli isolates from urine over the same time frame. Strikingly, all four ESBL-producing Morganella morganii isolates were resistant to cotrimoxazole, whereas the overall resistance rate to cotrimoxazole among non-ESBL $M$. morganii isolates over the same time frame was only $18.3 \%$. Susceptibility to the 7 - $\alpha$-substituted $\beta$-lactam cefoxitin was $100 \%$ (7/7) among the Proteus, $90.9 \%$ (40/44) among the Klebsiella, 82.6\% (138/167) among the E. coli and $75.0 \%$ (3/4) among the Serratia ESBL producers, whilst susceptibility to cefotetan was $100 \%(1 / 1)$ among the Proteus, $98.6 \%$ (71/72) among the E. coli and 93.3\% (28/30) among the Klebsiella ESBL-positive isolates tested for these antibiotics. Unfortunately, although cefotetan has better susceptibility rates, it is not currently available in the domestic German trade.

\section{Carbapenem-non-susceptible ESBL isolates}

Carbapenems are usually used for antibiotic treatment of infections with ESBL producers. Although most of the 


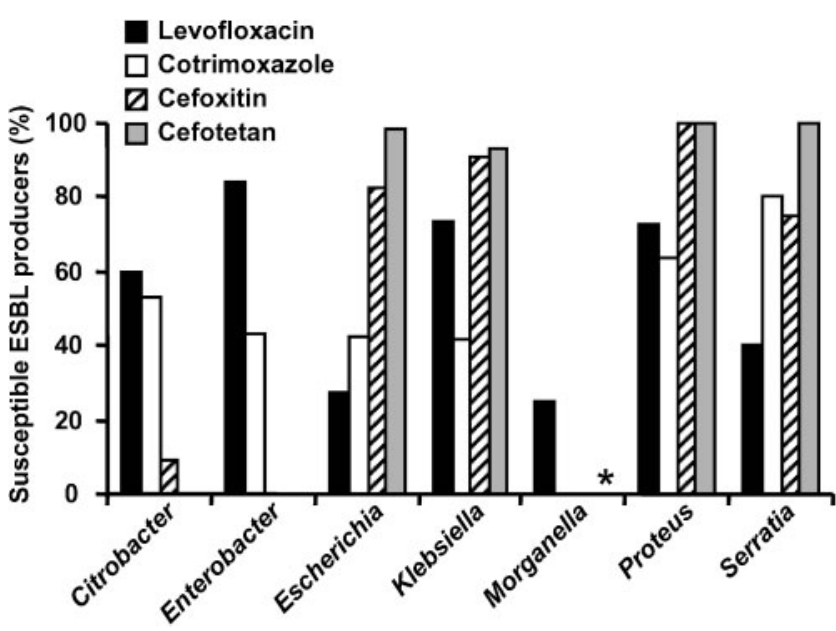

Fig. 2. Susceptibility testing of ESBL isolates. Percentage of ESBL producers per species susceptible to the indicated antibiotics. All of the ESBL-positive isolates were tested against levofloxacin and cotrimoxazole. Only selected isolates were tested against cefoxitin and cefotetan (cefoxitin: Citrobacter, $n=11$; Enterobacter, $n=47 ; \quad$ E. coli, $n=167 ;$ Klebsiella, $n=44$; Morganella, $n=2$; Proteus, $n=7$; Serratia, $n=4$; cefotetan: Citrobacter, $n=4$; Enterobacter, $n=8$; E. coli, $n=72$; Klebsiella, $n=30$; Proteus, $n=1$; Serratia, $n=3$ ). ${ }^{*}$, Not applicable (no Morganella isolate was tested).

ESBL-positive isolates in our hospital were susceptible to meropenem, we found that five K. pneumoniae isolates and one $E$. coli isolate, which together represented $1.6 \%$ of the total ESBL-producing strains, were of intermediate susceptibility (two K. pneumoniae, one E. coli isolate) or were resistant (three K. pneumoniae isolates) to meropenem, confirmed by a microbroth dilution assay and by Etest. The meropenem and imipenem MIC values of the carbapenemnon-susceptible K. pneumoniae isolates and their carbapenem-susceptible counterparts, as well as the resistance profile to other antibiotics and some information concerning patients $(\mathrm{A}-\mathrm{E})$ that the isolates were derived from, are given in Table 2.

Resistance to cefoxitin was observed in all isolates, with increased MIC values for imipenem and meropenem, whereas the carbapenem-susceptible counterparts (isolate 187 from patient A and isolate 46 from patient B) were susceptible to cefoxitin. Moreover, all carbapenem-nonsusceptible Klebsiella isolates exhibited intermediate susceptibility or resistance to colistin.

Strikingly, isolates 202 (patient A) and 201 (patient C) had the same antibiotic susceptibility profile and were isolated at an interval of 1 day, indicating a possible relatedness among these two strains. This assumption was confirmed by PCR analysis (Table 3 ) showing that both isolates carried the ESBL gene $b l a_{\mathrm{CTX}-\mathrm{M}-15}$ and the classical $b l a_{\mathrm{SHV}-1}$ gene and exhibited the same amino acid substitutions in the ompK36 gene, indicating that these two isolates were identical. However, there was no indication of the possible route of transmission from patient $\mathrm{A}$ (child) to patient $\mathrm{C}$ (adult), as these patients did not share the same ward and were not treated in the same operation area or other 'transfer areas' such as computed tomography at the same time.

Whereas most of the carbapenem-non-susceptible $K$. pneumoniae isolates with increased carbapenem MIC values only colonized patients $(\mathrm{A}-\mathrm{C}, \mathrm{E})$, one of the $K$. pneumoniae isolates (patient D) caused an infection. This patient was transferred by emergency to Germany by family members and admitted to our hospital after she had undergone several revision operations in Turkey because of a hip prosthesis infection due to ESBL-positive $K$. pneumoniae. Therefore, this isolate was probably imported to our hospital from Turkey.

We have documented that two ESBL-positive K. pneumoniae isolates (patients A and B) developed carbapenem resistance in vivo after treatment with meropenem for 60 days (patient $\mathrm{A}$ ) and 3 days after discontinuation of the meropenem-containing regime for 13 days (patient $\mathrm{B}$ ) (Table 2). Also patients $\mathrm{D}$ and $\mathrm{E}$ had received meropenem prior to the isolation of the carbapenem-non-susceptible ESBL Klebsiella, whereas patient $\mathrm{C}$ did not receive carbapenems as he had been admitted to our hospital 23 days before the detection of ESBL. Thus, in four of the five patients, antibiotic treatment with meropenem preceded the isolation of carbapenem-non-susceptible $K$. pneumoniae isolates. Moreover, two of these patients developed carbapenem resistance in vivo, indicating that antibiotic treatment of ESBL producers with carbapenems is not sufficient and, additionally, that carbapenems may select for resistance. It is being increasingly reported that carbapenem resistance in K. pneumoniae develops in strains producing plasmid-encoded AmpC $\beta$-lactamase or other broad-spectrum $\beta$-lactamases such as ESBLs due to the selection of mutants with reduced permeability to these drugs or, alternatively, carbapenemases (Jacoby et al., 2004; Kaczmarek et al., 2006; Mena et al., 2006; Queenan \& Bush, 2007; Yigit et al., 2001).

\section{Resistance gene location and transfer}

In order to analyse whether ESBLs were present in the carbapenem-non-susceptible $K$. pneumoniae isolates, PCR analyses of the strains using universal primers for detection and sequencing of $b l a_{\mathrm{TEM}}, b l a_{\mathrm{SHV}}$ and $b l a_{\mathrm{CTX}-\mathrm{M}}$ genes were performed and bla genes were identified in all isolates. Five isolates harboured a single ESBL gene $\left(b l_{\mathrm{TEM}-29}, n=1\right.$; $\left.b l a_{\mathrm{SHV}-2}, n=4\right)$. The remaining isolates contained the ESBL-type CTX-M-15 and a SHV-1 and/or TEM-1 $\beta$ lactamase (Table 3 ). The gene $b l a_{\mathrm{CTX}-\mathrm{M}-15}$ was found to be located on a plasmid of $\sim 40 \mathrm{~kb}$ in three clonally related $K$. pneumoniae isolates. In isolate $263, b l a_{\mathrm{TEM}-1}$ and $b l a_{\mathrm{CTX}-\mathrm{M}-}$ 15 were found on a $40 \mathrm{~kb}$ plasmid, whereas isolate 467 contained an $80 \mathrm{~kb}$ plasmid encoding $b l a_{\mathrm{SHV}-12}$ as well as $b l a_{\mathrm{CTX}-\mathrm{M}-15}$ (Table 3). Via conjugation in two isolates, the 
Table 2. Susceptibility patterns and sources of the carbapenem-non-susceptible ESBL-producing $K$. pneumoniae isolates and their susceptible counterparts

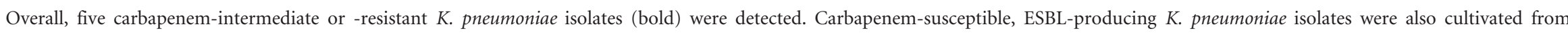

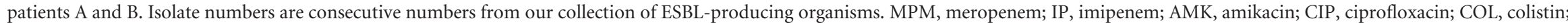
FOS, fosfomycin; FOX, cefoxitin; GEN, gentamicin; LEV, levofloxacin; OTE, oxytetracycline; SXT, sulfamethoxazole/trimethoprim; TOB, tobramycin.

\begin{tabular}{|c|c|c|c|c|c|c|c|c|}
\hline \multirow[t]{2}{*}{ Patient } & \multirow[t]{2}{*}{ Hospital ward } & \multirow{2}{*}{$\begin{array}{c}\text { Isolate } \\
\text { no. }\end{array}$} & \multirow{2}{*}{$\begin{array}{l}\text { MIC (MPM/IP; } \\
\left.\quad \mu \mathrm{g} \mathrm{ml}^{-1}\right)\end{array}$} & \multirow[t]{2}{*}{ Specimen } & \multirow{2}{*}{$\begin{array}{c}\text { Date of specimen } \\
\text { receipt }\end{array}$} & \multicolumn{3}{|c|}{ Antibiotic profile } \\
\hline & & & & & & Susceptible & Intermediate & Resistant \\
\hline \multirow[t]{2}{*}{ A } & Paediatric haematology ICU & 202 & $16 / 8$ & Stool & 23.01 .06 & GEN & OTE, AMK & $\begin{array}{l}\text { TOB, SXT, LEV, CIP, FOS, } \\
\text { COL, FOX }\end{array}$ \\
\hline & & 187 & $\leqslant 0.063 / 0.25$ & Stool & 5.12 .05 & GEN, COL, FOX & OTE, AMK & TOB, SXT, LEV, CIP, FOS \\
\hline \multirow[t]{4}{*}{ B } & Paediatric oncology & 51 & $8 / 4$ & Urine & 2.02 .04 & GEN, TOB, LEV, CIP, AMK & & SXT, FOX, OTE, FOS, COL \\
\hline & & 49 & $4 / 4$ & Vaginal swab & 28.01 .04 & GEN, TOB, LEV, CIP, AMK & & SXT, FOX, OTE, FOS, COL \\
\hline & & 52 & $4 / 2$ & Vaginal swab & 4.02 .04 & GEN, TOB, LEV, CIP, AMK & & SXT, FOX, OTE, FOS, COL \\
\hline & & 46 & $<0.063 / 0.5$ & Stool & 7.01 .04 & $\begin{array}{l}\text { GEN, TOB, LEV, CIP FOS, COL, } \\
\text { FOX, AMK }\end{array}$ & & SXT, OTE \\
\hline $\mathrm{C}$ & Haemato-oncology ICU & 201 & $16 / 8$ & Stool & 24.01 .06 & GEN & OTE, AMK & $\begin{array}{l}\text { TOB, SXT, LEV, CIP, FOS, } \\
\text { COL, FOX }\end{array}$ \\
\hline $\mathrm{D}$ & Orthopaedics & 263 & $32 / 64$ & Wound & 14.08 .06 & AMK & $\mathrm{COL}$ & $\begin{array}{l}\text { GEN, TOB, OTE, SXT, LEV, } \\
\text { CIP, FOS, FOX }\end{array}$ \\
\hline $\mathrm{E}$ & Paediatric haematology ICU & 467 & $8 / 4$ & Stool & 29.10 .07 & AMK & COL & $\begin{array}{l}\text { GEN, TOB, OTE, SXT, LEV, } \\
\text { CIP, FOS, FOX }\end{array}$ \\
\hline
\end{tabular}


Table 3. Resistance and changes in porin genes of the carbapenem-non-susceptible $K$. pneumoniae and $E$. coli isolates and their susceptible counterparts

The presence of ESBL resistance genes of all five carbapenem-non-susceptible K. pneumoniae isolates and one E. coli ESBL isolate was investigated by conventional PCR. Transfer experiments were performed with E. coli J53. Changes in porin genes (ompK35 and ompK36) in the K. pneumoniae strains were determined by sequence analyses. ESBL types are shown in italics, and carbapenem-non-susceptible isolates in bold. ND, Not determined; I-TGA/I-TAA, insertion resulting in the stop codon TGA or TAA, respectively; M-TGA, mutation resulting in the stop codon TGA.

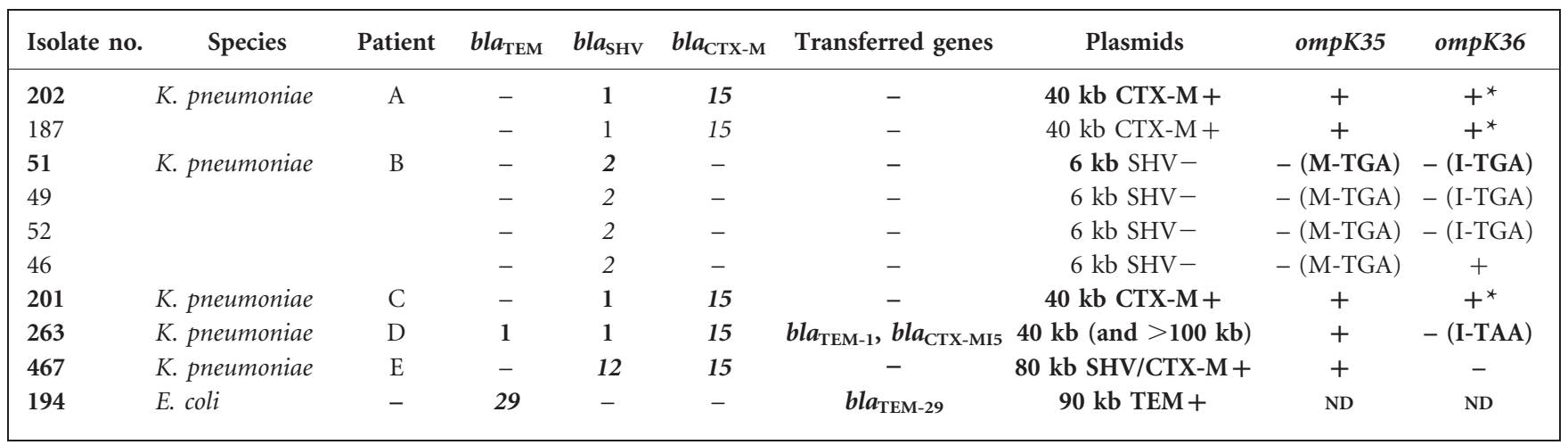

*Various amino acid substitutions.

resistance genes (isolate $194, b l a_{\mathrm{TEM}-29}$, and isolate 263, $\left.b l a_{\mathrm{TEM}-1}+b l a_{\mathrm{CTX}-\mathrm{M}-15}\right)$ could be transferred successfully in E. coli J53. The plasmid profiles of isolate 263 and its transconjugant showed a $40 \mathrm{~kb}$ plasmid and another weak plasmid band ( $>100 \mathrm{~kb}$ ), but Southern blot hybridization was not successful. The $b l a_{\mathrm{SHV}-2}$ gene of four clonally related $K$. pneumoniae isolates was not transferred and could not be identified on a plasmid by Southern blot hybridization.

As K. pneumoniae normally lacks a chromosomally encoded class C $\beta$-lactamase, acquisition of a plasmidencoded $b l a_{\mathrm{AmpC}}$ combined with porin loss or carbapenemases can confer resistance to carbapenems (Bradford et al., 1997). However, PCR analysis of all five carbapenem-nonsusceptible K. pneumoniae isolates for plasmid-borne ampC genes and relevant carbapenem-resistance genes ( $b l a_{\mathrm{KPC}}$, $b l a_{\mathrm{OXA}-23}, b l a_{\mathrm{OXA}-24}, b l a_{\mathrm{OXA}-48}, b l a_{\mathrm{OXA}-58}, b l a_{\mathrm{VIM}}, b l a_{\mathrm{IMP}}$, $b l a_{\mathrm{GIM}}, b l a_{\mathrm{SIM}}$ and $\left.b l a_{\mathrm{SPM}}\right)$ was negative, indicating the absence of AmpC $\beta$-lactamases or carbapenemases. Accordingly, analysis of a possible production of metallo$\beta$-lactamases of all carbapenem-non-susceptible $K$. pneumoniae isolates by Etest $\mathrm{MBL}$ yielded negative results. Additionally, a modified Hodge test, a phenotypic test with a $100 \%$ sensitivity in detecting KPC and which is also positive when other carbapenemases are present (Anderson et al., 2007), was negative for all five carbapenem-nonsusceptible $K$. pneumoniae isolates, indicating the absence of KPC and other carbapenemases.

The carbapenem-intermediate susceptible E. coli isolate (Table 3) with MIC values of $8 \mu \mathrm{g} \mathrm{ml}^{-1}$ for meropenem and $2 \mu \mathrm{g} \mathrm{ml}^{-1}$ for imipenem was isolated from urine in the paediatric haematology intensive care unit and displayed the ESBL phenotype of cefpodoxime MIC of $>8 \mu \mathrm{g} \mathrm{ml}^{-1}$, ceftazidime MIC of $>32 \mu \mathrm{g} \mathrm{ml}^{-1}$, inhibited by clavulanic acid: MIC ratio $\geqslant 8$, and ESBL genotype TEM-29. Actually, there are only a few reports worldwide describing this ESBL variant, which is characterized by a noticeable resistance pattern conferring resistance to ceftazidime but not to cefotaxime. Analysis of the chromosomal ampC promoter sequence of this isolate revealed mutations at position -42 of the $a m p C$ promoter sequence associated with a mutation at position -18 whereby two new alternative promoter boxes were generated. It is known that these changes result in high-level expression of chromosomal E. coli ampC and mediate cefoxitin resistance in E. coli (Caroff et al., 2000), suggesting that the cefoxitin resistance was due to the mutations of the chromosomal ampC promoter sequence. However, this suggestion could not be confirmed by agar disc diffusion testing for cefoxitin on Mueller-Hinton agar plates containing the AmpC inhibitor cloxacillin, as no increase in the cefoxitin inhibition zone diameter was observed when compared with testing on agar plates without the inhibitor. By contrast, meropenem MIC values were reduced in the presence of cloxacillin $\left(250 \mu \mathrm{g} \mathrm{ml}^{-1}\right)$ by two twofold dilutions (tested by Etest), indicating that the high-level expression of chromosomal E. coli ampC might contribute to carbapenem resistance, as it has previously been described in combination with porin loss (Livermore \& Woodford, 2006).

\section{Investigation of porins}

Loss of the two major porins, the outer-membrane proteins OmpK35 and/or OmpK36, has been associated with carbapenem resistance in K. pneumoniae (Kaczmarek et al., 2006; Martínez-Martínez et al., 1999). Moreover, the development of resistance to carbapenems in vivo in ESBL- 
positive $K$. pneumoniae isolates due to the selection of mutants with a lack of porin expression and reduced permeability to these drugs has been reported previously (Lee et al., 2007; Mena et al., 2006). In particular, the Cterminal $\mathrm{F}$ residue of OmpK36 is necessary for outermembrane expression (Struyve et al., 1991).

Sequence analysis of the coding region of the ompK35 gene in four clonally related $K$. pneumoniae isolates (isolates 46, 49, 51 and 52, all from patient B) revealed a change in position 135 from codon TGG to stop codon TGA (Table 3). Furthermore, three of these isolates that showed an increased MIC for carbapenems (isolates 49, 51 and 52) had an insertion of 8 bp (GCGCGGCG) in the ompK36 sequence resulting in a frameshift and generation of a stop codon (TGA) and a lack of the last 155 aa. These changes, combined with expression of the SHV-2 $\beta$ lactamase, might confer resistance to carbapenems and produce elevated MIC values to carbapenems, respectively, on these isolates as described previously (Crowley et al., 2002). By contrast, in the carbapenem-susceptible isolate 46, this frameshift was not found (Table 3), supporting a role for OmpK36 expression in carbapenem susceptibility.

In the ompK36 gene of isolate 263 (patient D), a single base-pair insertion was detected that led to a preliminary stop codon, TAA, resulting in a porin truncated by 196 aa. Hence inactivation of OmpK36 in isolate 263 was caused by interruption of the coding sequence by a single basepair insertion similar to a previous report describing the interruption of the coding sequence by an insertion sequence (Hernández-Allés et al., 1999). In isolate 467 (patient E), ompK35 but not ompK36 was amplified by PCR. The failure of ompK36 amplification in isolate 467 suggests either a deletion of part of the complete coding sequence or its interruption by an insertion sequence that was too large to be amplified by conventional PCR. The presence of CTX-M ESBL in isolates 263 (patient D) and 467 (patient E), along with inactivation of OmpK36, might contribute to the carbapenem resistance in these two isolates. Moreover, cefoxitin resistance in ESBLpositive $K$. pneumoniae has been attributed to porin loss, as the resistance persists when the plasmid is eliminated, whilst the plasmid itself does not express cefoxitin resistance when transferred to a new host (MartínezMartínez et al., 1996, 1999). Hence, cefoxitin resistance in isolates 51, 263 and 467 could be attributed to this mechanism.

The carbapenem-resistant K. pneumoniae isolates 202 (patient A) and 201 (patient C) exhibited the same amino acid substitutions in the ompK36 gene. However, these changes did not seem to be causative of the resistance to carbapenems, as this mutation was also found in a susceptible counterpart, isolate 187 (patient A). Hence we looked for other possible changes in the bacterial cell wall of isolates 202 and 201 that might contribute to the carbapenem resistance of these isolates.

\section{Identification of efflux pumps by SDS-PAGE and MS}

In order to analyse possible changes in the bacterial cell membrane of the carbapenem-non-susceptible K. pneumoniae isolates, outer-membrane extracts of these isolates were separated by SDS-PAGE and stained with Coomassie solution. Bands of proteins expressed at increased levels in the carbapenem-non-susceptible isolates or, alternatively, only in the carbapenem-susceptible counterparts were excised, digested and analysed by MS. As shown in Fig. 3, both isolate 202 (patient A, lane 1) and isolate 201 (patient $\mathrm{C}$, lane 7) expressed all of the components of a tripartite AcrAB-TolC efflux pump (Bornet et al., 2003; Fernandez-Recio et al., 2004; Hu et al., 2007), namely the outer-membrane channel TolC, the trimeric inner-membrane proton antiporter $A c r B$ and the periplasmic membrane fusion protein AcrA, indicating that an efflux mechanism might contribute to the resistance to carbapenems in these two isolates. Accordingly, by MS analysis, these three proteins were not detectable in isolate 187 (patient A, lane 2), the carbapenem-susceptible counterpart to isolate 202. Moreover, isolates 202 and 201 both exhibited a reduction in meropenem MICs (tested by Etest) by one twofold dilution in the presence of the efflux pump inhibitors phenylalanine arginine $\beta$-naphthylamide $(\mathrm{PA} \beta \mathrm{N}$, $70 \mu \mathrm{M})$ and carbonyl cyanide 4-trifluoromethoxyphenylhydrazone (FCCP, $100 \mu \mathrm{M}$ ), respectively, which is closely related to the more common efflux pump inhibitor carbonyl cyanide $m$-chlorophenylhydrazone. Although the mechanisms responsible for increasing the K. pneumoniae MIC to a rather high level remain unclear, it is possible that the efflux pump system might have contributed to further increasing the meropenem MICs in these two isolates. By contrast, when Etests were performed with isolates 51, 263 and 467, no synergistic inhibitory effect between meropenem and the efflux pump inhibitors was observed, suggesting that efflux pumps do not play a role in the carbapenem resistance of these isolates. This was in accordance with the lack of efflux pumps in these isolates demonstrated by MS.

There is a report describing efflux pumps as the causative mechanism for multi-resistant K. pneumoniae isolates from Turkey (Hasdemir et al., 2004). Expression of this resistance-nodulation-cell division (RND) efflux system might also contribute to the concomitant resistance to cefoxitin of these two isolates as reported for RND-family efflux pumps in clinical isolates of Bacteroides fragilis (Pumbwe et al., 2006), as we observed a noticeable increase in the cefoxitin inhibition zone diameter by $8 \mathrm{~mm}$ each in the presence of the efflux pump inhibitors PA $\beta \mathrm{N}$ and FCCP. By contrast, a synergistic inhibitory effect of cefoxitin and the inhibitors was not observed in isolates 51, 263 and 467, suggesting that mechanisms other than efflux pumps are responsible for cefoxitin resistance in these isolates.

Unfortunately, we were not able to differentially detect OmpK35, OmpK36 and OmpA of the K. pneumoniae 


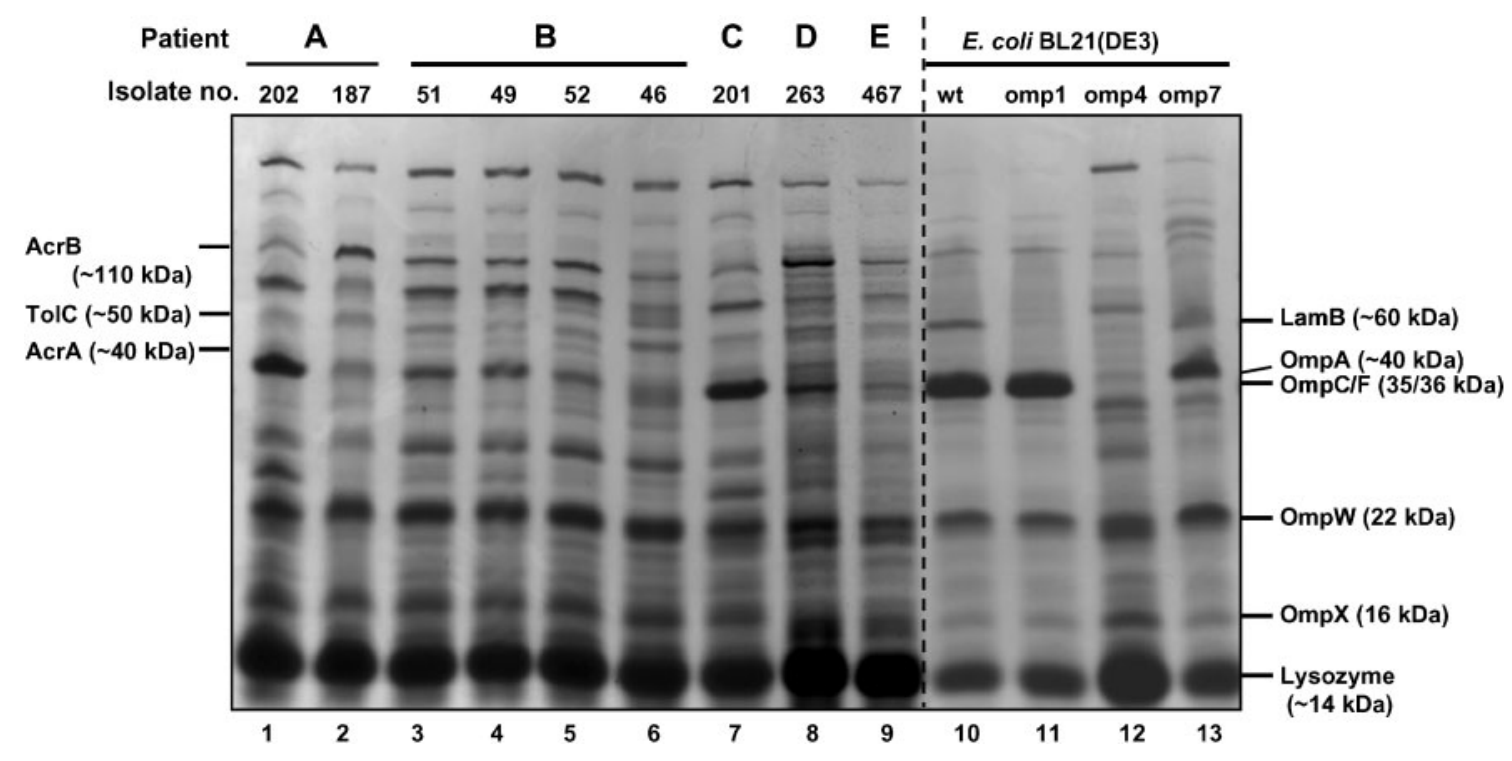

Fig. 3. Coomassie-stained SDS-polyacrylamide gel of outer-membrane proteins. The outer-membrane proteins of the nine meropenem-resistant or -intermediate ESBL-producing $K$. pneumoniae isolates and their meropenem-susceptible counterparts isolated from patients A-E (see Table 2) were separated by SDS-PAGE and 31 single bands were analysed by MS. As a control, E. coli BL21(DE3) wild-type (wt) (lane 10) and several deletion mutants (Prilipov et al., 1998) of this strain [omp1=BL21(DE3) $\Delta / a m B$; omp4=BL21(DE3) $\Delta / a m B$ ompF: :Tn5 $\Delta$ ompA; omp7=BL21(DE3) $\Delta / a m B$ ompF: :Tn5 $\Delta$ ompC; lanes $11-13$, respectively] were used. The corresponding outer-membrane proteins of these strains are indicated.

isolates from the SDS-PAGE analysis as these outermembrane proteins co-migrated. The E. coli OmpC (homologous to OmpK35), OmpF (homologous to OmpK36) and OmpA also co-migrated. Co-migration of these proteins was confirmed by immunoblot analysis with specific antisera against OmpC/F and OmpA (data not shown).

In summary, we have reported here the emergence of five sporadic carbapenem-non-susceptible, ESBL-positive $K$. pneumoniae isolates, two of which developed carbapenem resistance in vivo under a meropenem-containing regime, and one $E$. coli isolate. The mechanism of carbapenem resistance in three of the $K$. pneumoniae isolates was loss of porins essential for uptake of antibiotics. However, the mechanisms responsible for carbapenem resistance of the other two $K$. pneumoniae isolates remain unclear. One could speculate that post-translational changes in the OmpK36 protein leading to loss of porin or loss of function were causative in increasing the meropenem MICs to a rather high level, and it is possible that the overexpression of an efflux pump system in these two isolates might have contributed towards further increasing the meropenem MICs. Neither an outbreak nor transmission to other patients was observed. However, it cannot be excluded that the development of carbapenem resistance is largely underestimated as ESBLs are not currently systematically explored for intestinal colonization. Therefore, from our study and recent studies reporting the emergence of ESBL-positive isolates resistant to carbapenems (Lee et al., 2007; Liu et al., 2008; Mena et al., 2006), we conclude that medical professionals should be aware of these isolates, should continue strict hygiene procedures and, additionally, should implement an ESBL screening system, in particular for faecal carriage on haemato-oncological wards with increased use of carbapenems, in order to prevent possible outbreaks caused by these multi-resistant organisms.

\section{ACKNOWLEDGEMENTS}

The authors thank Matthias Marschal for help with the statistical analyses and Sina Bäßler, Claudia Glunz and Silke Toll for excellent technical assistance.

\section{REFERENCES}

Anderson, K. F., Lonsway, D. R., Rasheed, J. K., Biddle, J., Jensen, B., McDougal, L. K., Carey, R. B., Thompson, A., Stocker, S. \& other authors (2007). Evaluation of methods to identify the Klebsiella pneumoniae carbapenemase in Enterobacteriaceae. J Clin Microbiol 45, 2723-2725.

Barjaktarovic, Z., Nordheim, A., Lamkemeyer, T., Fladerer, C., Madlung, J. \& Hampp, R. (2007). Time-course of changes in amounts of specific proteins upon exposure to hyper-g, 2-D clinorotation, and 3-D random positioning of Arabidopsis cell cultures. J Exp Bot 58, 4357-4363.

Ben-Ami, R., Schwaber, M. J., Navon-Venezia, S., Schwartz, D., Giladi, M., Chmelnitsky, I., Leavitt, A. \& Carmeli, Y. (2006). Influx of extended-spectrum $\beta$-lactamase-producing Enterobacteriaceae into the hospital. Clin Infect Dis 42, 925-934. 
Bornet, C., Chollet, R., Mallea, M., Chevalier, J., Davin-Regli, A., Pages, J. M. \& Bollet, C. (2003). Imipenem and expression of multidrug efflux pump in Enterobacter aerogenes. Biochem Biophys Res Commun 301, 985-990.

Bradford, P. A., Urban, C., Mariano, N., Projan, S. J., Rahal, J. J. \& Bush, K. (1997). Imipenem resistance in Klebsiella pneumoniae is associated with the combination of ACT-1, a plasmid-mediated AmpC $\beta$-lactamase, and the loss of an outer membrane protein. Antimicrob Agents Chemother 41, 563-569.

Caroff, N., Espaze, E., Gautreau, D., Richet, H. \& Reynaud, A. (2000). Analysis of the effects of -42 and -32 ampC promoter mutations in clinical isolates of Escherichia coli hyperproducing AmpC. J Antimicrob Chemother 45, 783-788.

Castillo Garcia, F. J., Seral García, C., Pardos De la Gandara, M., Millán Lou, M. I. \& Pitart Ferré, C. (2007). Prevalence of fecal carriage of ESBL-producing Enterobacteriaceae in hospitalized and ambulatory patients during two non-outbreak periods. Eur J Clin Microbiol Infect Dis 26, 77-78.

CLSI (2008). Performance Standards for Antimicrobial Susceptibility Testing, 18th Informational Supplement, M100-S18. Wayne, PA: Clinical and Laboratory Standards Institute.

Crowley, B., Benedi, V. J. \& Domenech-Sanchez, A. (2002). Expression of SHV-2 $\beta$-lactamase and of reduced amounts of OmpK36 porin in Klebsiella pneumoniae results in increased resistance to cephalosporins and carbapenems. Antimicrob Agents Chemother 46, 3679-3682.

Fernandez-Recio, J., Walas, F., Federici, L., Venkatesh, P. J., Bavro, V. N., Miguel, R. N., Mizuguchi, K. \& Luisi, B. (2004). A model of a transmembrane drug-efflux pump from Gram-negative bacteria. FEBS Lett 578, 5-9.

Grimm, V., Ezaki, S., Susa, M., Knabbe, C., Schmid, R. D. \& Bachmann, T. T. (2004). Use of DNA microarrays for rapid genotyping of TEM beta-lactamases that confer resistance. $J$ Clin Microbiol 42, 3766-3774.

Hasdemir, U. O., Chevalier, J., Nordmann, P. \& Pages, J. M. (2004). Detection and prevalence of active drug efflux mechanism in various multidrug-resistant Klebsiella pneumoniae strains from Turkey. J Clin Microbiol 42, 2701-2706.

Hernández-Allés, S., Benedí, V. J., Martínez-Martínez, L., Pascual, A., Aguilar, A., Tomás, J. M. \& Albertí, S. (1999). Development of resistance during antimicrobial therapy caused by insertion sequence interruption of porin genes. Antimicrob Agents Chemother 43, $937-$ 939.

Hu, W. S., Yao, S. M., Fung, C. P., Hsieh, Y. P., Liu, C. P. \& Lin, J. F. (2007). An OXA-66/OXA-51-like carbapenemase and possibly an efflux pump are associated with resistance to imipenem in Acinetobacter baumannii. Antimicrob Agents Chemother 51, 38443852 .

Jacoby, G. A., Mills, D. M. \& Chow, N. (2004). Role of $\beta$-lactamases and porins in resistance to ertapenem and other $\beta$-lactams in Klebsiella pneumoniae. Antimicrob Agents Chemother 48, 3203-3206.

Kaczmarek, F. M., Dib-Hajj, F., Shang, W. \& Gootz, T. D. (2006). High-level carbapenem resistance in a Klebsiella pneumoniae clinical isolate is due to the combination of $b l a_{\mathrm{ACT}-1} \beta$-lactamase production, porin OmpK35/36 insertional inactivation, and down-regulation of the phosphate transport porin PhoE. Antimicrob Agents Chemother 50, 3396-3406.

Lee, C. H., Chu, C., Liu, J. W., Chen, Y. S., Chiu, C. J. \& Su, L. H. (2007). Collateral damage of flomoxef therapy: in vivo development of porin deficiency and acquisition of $b l a_{\mathrm{DHA}-1}$ leading to ertapenem resistance in a clinical isolate of Klebsiella pneumoniae producing CTX-M-3 and SHV-5 $\beta$-lactamases. J Antimicrob Chemother 60, 410-413.
Liu, Y., Lamkemeyer, T., Jakob, A., Mi, G., Zhang, F., Nordheim, A. \& Hochholdinger, F. (2006). Comparative proteome analyses of maize (Zea mays L.) primary roots prior to lateral root initiation reveal differential protein expression in the lateral root initiation mutant rum 1. Proteomics 6, 4300-4308.

Liu, Y. F., Yan, J. J., Ko, W. C., Tsai, S. H. \& Wu, J. J. (2008). Characterization of carbapenem-non-susceptible Escherichia coli isolates from a university hospital in Taiwan. $J$ Antimicrob Chemother 61, 1020-1023.

Livermore, D. M. \& Woodford, N. (2006). The $\beta$-lactamase threat in Enterobacteriaceae, Pseudomonas and Acinetobacter. Trends Microbiol 14, 413-420.

Macrina, F. L., Kopecko, D. J., Jones, K. R., Ayers, D. J. \& McCowen, S. M. (1978). A multiple plasmid-containing Escherichia coli strain: convenient source of size reference plasmid molecules. Plasmid 1, 417-420.

Martínez-Martínez, L., Hernández-Allés, S., Albertí, S., Tomás, J. M., Benedi, V. J. \& Jacoby, G. A. (1996). In vivo selection of porindeficient mutants of Klebsiella pneumoniae with increased resistance to cefoxitin and expanded-spectrum-cephalosporins. Antimicrob Agents Chemother 40, 342-348.

Martínez-Martínez, L., Pascual, A., Hernández-Allés, S., AlvarezDíaz, D., Suárez, A. I., Tran, J., Benedí, V. J. \& Jacoby, G. A. (1999). Roles of $\beta$-lactamases and porins in activities of carbapenems and cephalosporins against Klebsiella pneumoniae. Antimicrob Agents Chemother 43, 1669-1673.

Mena, A., Plasencia, V., Garcia, L., Hidalgo, O., Ayestarán, J. I., Alberti, S., Borrell, N., Pérez, J. L. \& Oliver, A. (2006). Characterization of a large outbreak by CTX-M-1-producing Klebsiella pneumoniae and mechanisms leading to in vivo carbapenem resistance development. J Clin Microbiol 44, 2831-2837.

Mendes, R. E., Kiyota, K. A., Monteiro, J., Castanheira, M., Andrade, S. S., Gales, A. C., Pignatari, A. C. \& Tufik, S. (2007). Rapid detection and identification of metallo- $\beta$-lactamase-encoding genes by multiplex real-time PCR assay and melt curve analysis. J Clin Microbiol 45, 544-547.

Mulvey, M. R., Bryce, E., Boyd, D. A., Ofner-Agostini, M., Land, A. M., Simor, A. E. \& Paton, S. (2005). Molecular characterization of cefoxitin-resistant Escherichia coli from Canadian hospitals. Antimicrob Agents Chemother 49, 358-365.

Osborn, M. J., Gander, J. E., Parisi, E. \& Carson, J. (1972). Mechanism of assembly of the outer membrane of Salmonella typhimurium. Isolation and characterization of cytoplasmic and outer membrane. J Biol Chem 247, 3962-3972.

Oteo, J., Navarro, C., Cercenado, E., Delgado-Iribarren, A., Wilhelmi, I., Orden, B., García, C., Miguelañez, S., Pérez-Vázquez, M. \& other authors (2006). Spread of Escherichia coli strains with high-level cefotaxime and ceftazidime resistance between the community, longterm care facilities, and hospital institutions. J Clin Microbiol 44, 23592366.

Paterson, D. L., Hujer, K. M., Hujer, A. M., Yeiser, B., Bonomo, M. D., Rice, L. B., Bonomo, R. A. \& International Klebsiella Study Group (2003). Extended-spectrum $\beta$-lactamases in Klebsiella pneumoniae bloodstream isolates from seven countries: dominance and widespread prevalence of SHV- and CTX-M-type $\beta$-lactamases. Antimicrob Agents Chemother 47, 3554-3560.

Pérez-Pérez, F. J. \& Hanson, N. D. (2002). Detection of plasmidmediated AmpC $\beta$-lactamase genes in clinical isolates by using multiplex PCR. J Clin Microbiol 40, 2153-2162.

Perkins, D. N., Pappin, D. J., Creasy, D. M. \& Cottrell, J. S. (1999). Probability-based protein identification by searching sequence databases using mass spectrometry data. Electrophoresis 20, 35513567. 
Pitout, J. D., Nordmann, P., Laupland, K. B. \& Poirel, L. (2005). Emergence of Enterobacteriaceae producing extended-spectrum $\beta$ lactamases (ESBLs) in the community. J Antimicrob Chemother 56, 52-59.

Prilipov, A., Phale, P. S., Van Gelder, P., Rosenbusch, J. P. \& Koebnik, R. (1998). Coupling site-directed mutagenesis with highlevel expression: large scale production of mutant porins from E. coli. FEMS Microbiol Lett 163, 65-72.

Pumbwe, L., Glass, D. \& Wexler, H. M. (2006). Efflux pump overexpression in multiple-antibiotic-resistant mutants of Bacteroides fragilis. Antimicrob Agents Chemother 50, 3150-3153.

Queenan, A. M. \& Bush, K. (2007). Carbapenemases: the versatile $\beta$ lactamases. Clin Microbiol Rev 20, 440-458.

Ramphal, R. \& Ambrose, P. G. (2006). Extended-spectrum $\beta$ lactamases and clinical outcomes: current data. Clin Infect Dis 42 (Suppl. 4), S164-S172.

Schägger, H. \& von Jagow, G. (1987). Tricine-sodium dodecyl sulfate-polyacrylamide gel electrophoresis for the separation of proteins in the range from 1 to $100 \mathrm{kDa}$. Anal Biochem 166 , 368-379.

Struyve, M., Moons, M. \& Tommassen, J. (1991). Carboxy-terminal phenylalanine is essential for the correct assembly of a bacterial outer membrane protein. J Mol Biol 218, 141-148.
Sturenburg, E. \& Mack, D. (2003). Extended-spectrum $\beta$-lactamases: implications for the clinical microbiology laboratory, therapy, and infection control. J Infect 47, 273-295.

Tonkic, M., Goic-Barisic, I. \& Punda-Polic, V. (2005). Prevalence and antimicrobial resistance of extended-spectrum $\beta$-lactamases-producing Escherichia coli and Klebsiella pneumoniae strains isolated in a university hospital in Split, Croatia. Int Microbiol 8, 119-124.

Valverde, A., Grill, F., Coque, T. M., Pintado, V., Baquero, F., Canton, R. \& Cobo, J. (2008). High rate of intestinal colonization with extended spectrum $\beta$-lactamase-producing organisms in household contacts of infected community patients. J Clin Microbiol 46, 2796-2799.

Walsh, T. R., Bolmstrom, A., Qwarnstrom, A. \& Gales, A. (2002). Evaluation of a new Etest for detecting metallo- $\beta$-lactamases in routine clinical testing. J Clin Microbiol 40, 2755-2759.

Woodford, N., Tierno, P. M., Jr, Young, K., Tysall, L., Palepou, M. F., Ward, E., Painter, R. E., Suber, D. F., Shungu, D. \& other authors (2004). Outbreak of Klebsiella pneumoniae producing a new carbapenem-hydrolyzing class A $\beta$-lactamase, KPC-3, in a New York Medical Center. Antimicrob Agents Chemother 48, 4793-4799.

Yigit, H., Queenan, A. M., Anderson, G. J., Domenech-Sanchez, A., Biddle, J. W., Steward, C. D., Alberti, S., Bush, K. \& Tenover, F. C. (2001). Novel carbapenem-hydrolyzing $\beta$-lactamase, KPC-1, from a carbapenem-resistant strain of Klebsiella pneumoniae. Antimicrob Agents Chemother 45, 1151-1161. 